\title{
On the nature of the galaxy NGC 5666
}

\author{
C. J. Donzelli ${ }^{1, \star}$ and E. Davoust ${ }^{2}$ \\ 1 IATE, Observatorio Astronómico, Universidad Nacional de Córdoba, \& CONICET, Laprida 854, 5000, Córdoba, Argentina \\ 2 UMR 5572, Observatoire Midi-Pyrénées, 14 avenue E. Belin, 31400 Toulouse, France
}

Received 8 April 2003 / Accepted 2 July 2003

\begin{abstract}
We present $B$ and $R$ CCD images and optical spectroscopy in the range 3700-8900 $\AA$ for the galaxy NGC 5666, which until now was considered as a low-luminosity elliptical. The high-resolution images show a conspicuous spiral pattern which is limited to the inner region $\left(r \leq 5^{\prime \prime}\right.$, or $1 / 6$ of the isophotal radius) and a faint (presumably tidal) outer feature dotted with small knots resembling star clusters or tidal dwarf galaxies.

The patchy spiral structure and the luminosity profiles suggest a late-type morphological type, but the disk is of high central surface brightness and the bulge weak and very extended. The nuclear spectrum reveals typical emission lines found in late-type galaxies, but the underlying nuclear stellar population and continuum only match that of an elliptical galaxy combined with a young $(<1 \mathrm{Gyr})$ stellar population. These and other properties of the galaxy suggest that this could be a minor merger, at an intermediate stage, between a gas-rich dwarf and a small early-type disk galaxy. If this is the case, the galaxy NGC 5666 will provide important constraints to the theory of hierarchical galaxy formation.
\end{abstract}

Key words. galaxies: photometry

\section{Introduction}

NGC 5666 was first thought to be an elliptical galaxy. It was classified as compact by Nilson (1973) and as elliptical by Djorgovski (1985) and Lake et al. (1987) (hereafter LSG87) who found a luminosity profile which was well fitted by the $r^{1 / 4}$ law. The latter authors also found a total integrated color $(B-V)=0.9$, which is typical of elliptical galaxies.

Since elliptical galaxies are generally devoid of interstellar gas and thus of star formation, this galaxy attracted considerable attention when it was discovered that it had detectable amounts of HI (Peterson 1979; Lake \& Schommer 1984) and of CO (Gordon 1991; Lees et al. 1991). This cold interstellar gas was then mapped in details (see Sect. 5.4) and the galaxy was included in most discussions on the origin of the gas and on star formation in early-type galaxies, which remains an important issue to this day.

We first obtained high-resolution CCD images of this galaxy for a survey of IRAS-bright elliptical galaxies, and, to our surprise, these images clearly showed spiral structure. The fact that this is not an elliptical galaxy was confirmed by Prugniel \& Heraudeau (1998), who, on the basis of aperture

Send offprint requests to: C. J. Donzelli, e-mail: charly@oac.uncor.edu

* Visiting Astronomer at Complejo Astronómico el Leoncito (CASLEO) Observatory. CASLEO is operated under agreement between the Consejo Nacional de investigaciones Científicas y Técnicas de la República Argentina and the National Universities of La Plata, Córdoba and San Juan. photometry measurements, give the galaxy a photometric type of 7.7 (although with a quality of 6 , the lowest acceptable), which corresponds Scd in the classical morphological classification.

The goal of this paper is to present new photometric and spectroscopic data on this galaxy in order to reveal its true nature. If this galaxy had indeed been misclassified, it muddles the issue on the nature of gas-rich ellipticals, and has to be removed from the corresponding studies. Furthermore, revealing its true nature may provide useful clues on the formation of galaxies. The paper is organized as follows: In Sect. 2 we describe the observations and data reductions. The model for the luminosity distribution determined in Sect. 3 is subtracted from the original images to provide a detailed morphological description of the galaxy in Sect. 4. Our optical spectra and data on $\mathrm{HI}$ and $\mathrm{CO}$ from the literature are used to discuss the gaseous and stellar content of the galaxy in Sect. 5. We interpret our findings in Sect. 6, and a conclusion is given in Sect. 7.

\section{Observations and data reduction}

$B$ and $R$ images were taken at the Canada-France-Hawai 3.6 meter telescope in July 1992. A $640 \times 1024$ RCA CCD camera was used, with a pixel size of $0.108^{\prime \prime}$ and covering a $69^{\prime \prime} \times 110^{\prime \prime}$ area. The night was photometric and seeing conditions were excellent, namely $F W H M=0.5^{\prime \prime}$ and $0.4^{\prime \prime}$ in the $B$ and $R$ bands respectively. The log is summarized in Table 1.

The images were corrected for bias and flat-fielded using standard routines. Sky subtraction was carefully performed by 
Table 1. Photometric and spectroscopic observations.

\begin{tabular}{|c|c|c|c|c|c|}
\hline \multicolumn{3}{|c|}{ Images } & \multicolumn{3}{|c|}{ Spectra } \\
\hline Filter & Exp. (s) & Date & Range $(\AA)$ & Exp. (s) & Date \\
\hline$B$ & 1200 & 31.07 .92 & $3600-7000$ & $3 \times 900$ & 2.04 .02 \\
\hline$R$ & 600 & 31.07 .92 & 5700-9000 & $3 \times 900$ & 4.04 .02 \\
\hline
\end{tabular}

averaging mean values of the sky on the borders of the images to avoid galaxy contamination.

The photometric calibration was made using published aperture photometry (listed in the HYPERCAT Catalogue, see Prugniel \& Heraudeau 1998) for NGC 5666 and six other galaxies observed the same night, The final calibration was achieved by averaging individual zero points obtained for the seven observed galaxies. The estimated errors are 0.07 mag in $B$ and 0.05 mag in $R$.

Long-slit spectroscopy of NGC 5666 was carried out using a Tex $1024^{2} \mathrm{CCD}$ attached to the REOSC spectrograph on the 2.15 meter Ritchey-Chrétien telescope at the CASLEO Observatory in April 2002. Two sets of three 900-second exposures were obtained in the 3700-7000 $\AA$ and in the 5700$9000 \AA$ ranges respectively. The galaxy was observed using a $2.5^{\prime}$ long and $2.5^{\prime \prime}$ wide slit centered on the nucleus and oriented in the $\mathrm{E}-\mathrm{W}$ direction. We used a 300 -line $\mathrm{mm}^{-1}$ grating resulting in a spectroscopic resolution of $\sim 4 \AA$. The log of observations is listed in Table 1.

The spectra were reduced with the standard IRAF routines, they were wavelength-calibrated using $\mathrm{He}-\mathrm{Ne}-\mathrm{Ar}$ comparison spectra. The flux calibration was done with standard stars taken from the Catalogue of Southern Spectrophotometric Standards (Stone \& Baldwin 1983). The estimated rms uncertainty is 0.15 mag for residuals in fitting the system sensitivity function. The final spectrum was corrected for galactic absorption using the $A_{B}$ value obtained by Schlegel et al. (1998).

\section{3. $B$ and $R$ luminosity profiles}

The photometric profiles are presented in Fig. 1. They were obtained using the ELLIPSE routine within STSDAS (Jedrzejewski 1987). The clumpy structure in the inner 5" forced us to mask several HII regions to obtain convergence of the algorithm.

The inner region of the galaxy consists of a small central bulge and of a plateau which corresponds to the spiral structure shown in Fig. 2. The color profile shows that this inner region is very red, and reaches $(B-R)=1.4$ at the center. This could mean that the inner region is heavily obscured by dust, and that the bulge is in fact mostly hidden (see Sect. 5.3).

The outer profiles have two slopes, suggestive of a disk and of a halo of accreted luminous matter. A faint arm or tail at a radius of about $15^{\prime \prime}$, visible in Fig. 3, appears as a slight bump in the profiles.

In order to compute standard photometric parameters of the galaxy, we assume that it is composed of an $R^{1 / 4}$ bulge and an exponential disk. A halo component was not taken into account since the larger number of free parameters would prevent the

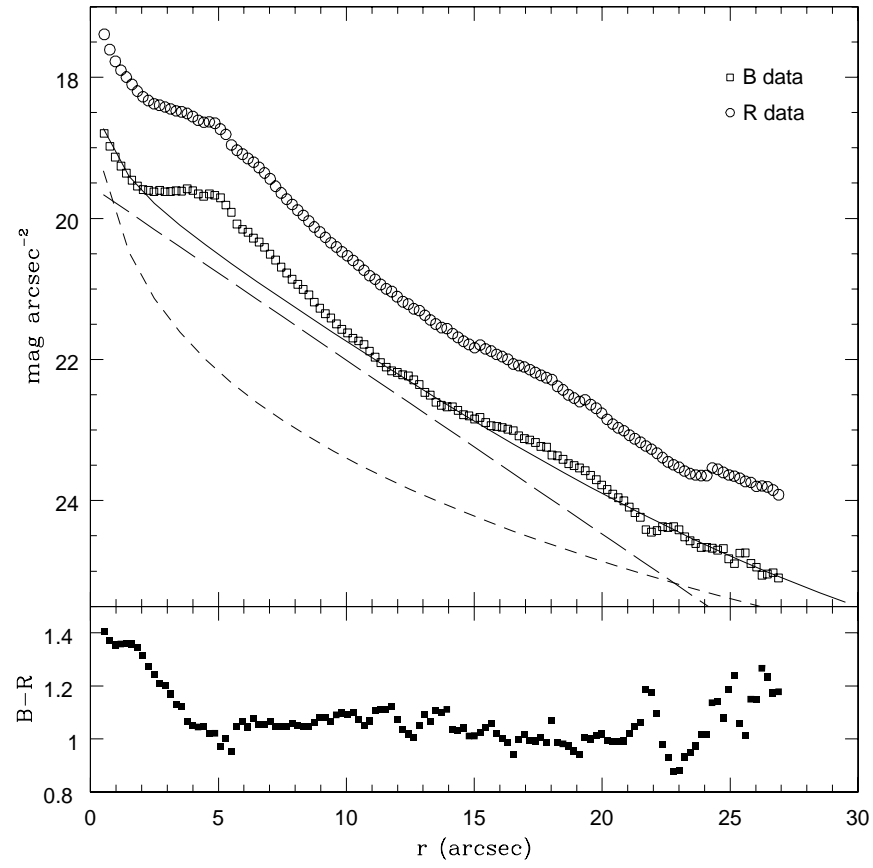

Fig. 1. $B, R$ and $(B-R)$ profiles for NGC 5666. The decomposition into bulge (short dashed) and disk (long dashed) components is shown for the $B$ data only.

convergence of the fitting algorithm. The functional form of each fitted component is as follows:

$I(r)=I_{\mathrm{e}} \exp \left(-7.688 *\left(\left(r / r_{\mathrm{e}}\right)^{.25}-1\right)\right)$

$I(r)=I_{\mathrm{d}} \exp \left(-r / d_{1}\right)$

for the bulge and the disk, respectively.

In the above equations $I_{\mathrm{e}}$ is the intensity at the effective radius $r_{\mathrm{e}}, I_{\mathrm{d}}$ is the central intensity and $d_{1}$ the scale length of the disk component.

We also tried the Sérsic law (Sérsic 1968) to fit the bulge component, namely:

$I(r)=I_{0} \exp (-r / \alpha)^{N}$.

The free parameter $N$ is useful when one looks for deviations from the $R^{1 / 4}$ law. However, our fit to the bulge is not improved with this law.

In order to obtain the above parameters we followed the method described by Schombert \& Bothun (1987) using the NFIT routine implemented in STSDAS. The routine must be provided with appropriate initial parameters to begin the fit. These parameters are generally guessed from visual inspection of the profile, because the inner region is bulge dominated and the outermost region is disk dominated. However, as can be seen in Fig. 1, this is not the case. Therefore we used an extensive set of initial parameters for both bulge and disk and we obtained the following results:

a) the final parameters are almost independent of the initial ones,

b) the difference between the final parameters from different tries arose from the fitting interval, which is the main source of error. 
Table 2. Photometric parameters.

\begin{tabular}{|c|c|c|c|c|c|c|c|c|}
\hline \multirow[b]{2}{*}{ Filter } & \multirow[b]{2}{*}{$m_{\mathrm{T}}$} & \multicolumn{3}{|c|}{ bulge } & \multicolumn{3}{|c|}{ disk } & \multirow[b]{2}{*}{$\mathrm{B} / \mathrm{D}$} \\
\hline & & $m_{\mathrm{e}}^{a}$ & $r_{\mathrm{e}}^{b}$ & $M_{\mathrm{T}}^{c}$ & $m_{0}^{a}$ & $d_{1}^{b}$ & $M_{\mathrm{T}}^{d}$ & \\
\hline$B$ & 13.75 & $24.00 \pm 0.5$ & $13 \pm 2$ & -17.55 & $19.54 \pm 0.2$ & $4 \pm 1$ & -18.17 & 0.6 \\
\hline$R$ & 12.72 & $22.70 \pm 0.4$ & $13 \pm 2$ & -18.80 & $18.07 \pm 0.2$ & $4 \pm 1$ & -19.35 & 0.6 \\
\hline
\end{tabular}

\footnotetext{
${ }^{a}$ In units of mag $\operatorname{arcsec}^{-2}$.

${ }^{b}$ In units of $"$.

${ }^{c} R^{1 / 4}$ profile integration.

${ }^{d}$ Exponential profile integration.
}

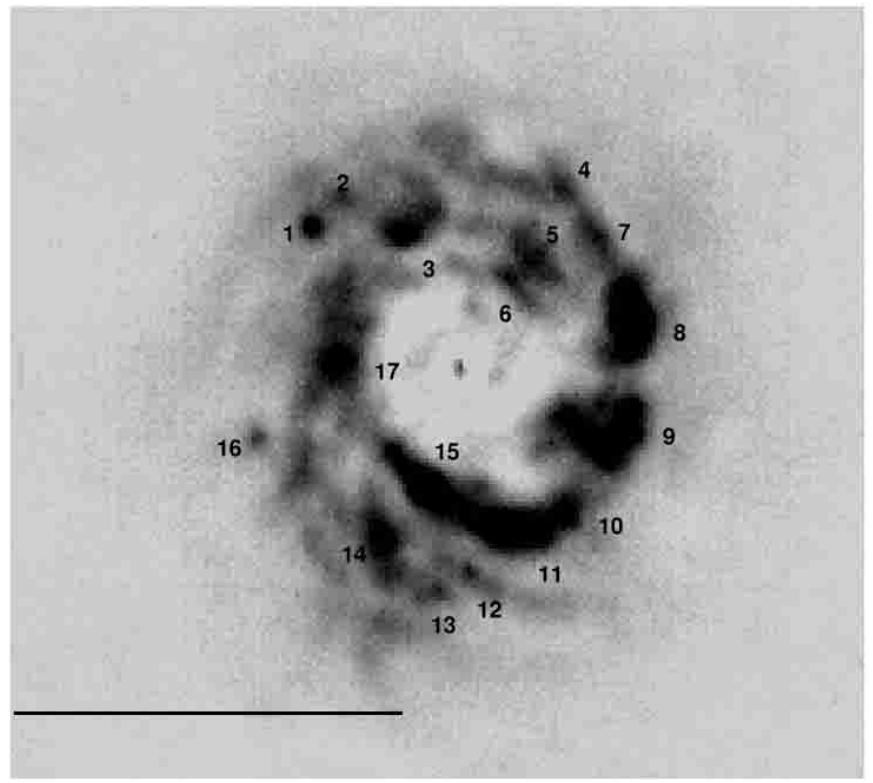

Fig. 2. $B$ image of NGC 5666 minus bulge+disk model galaxy. North is on top and East to the left. The lower left bar is $10^{\prime \prime}(=1.5 \mathrm{kpc})$ long.

Figure 1 shows the components fitted to the $B$ luminosity profile. We did not use the regions $2^{\prime \prime} \leq r \leq 12^{\prime \prime}$ and $15^{\prime \prime} \leq r \leq$ $22^{\prime \prime}$ in the fit. These "humps" in the luminosity profiles, which are more conspicuous in the $B$ band, are caused by strong HII regions and a faint outer arm/tail respectively (see Sect. 4).

The total luminosity of both components in both filters was computed using the derived photometric parameters and integrating Eqs. (1) and (2) as follows:

$L=\int_{0}^{\infty} I(r) 2 \pi r \mathrm{~d} r$

which yields:

$L_{\text {bulge }}=7.21 \pi I_{\mathrm{e}} r_{\mathrm{e}}^{2}$

$L_{\text {disk }}=2 \pi I_{0} d_{1}^{2}$

for the bulge and the disk respectively.

The total magnitude of the galaxy was estimated by two independent methods. The first one is the integration of the intensity on the images in a series of simulated concentric apertures. The second method consisted in integrating the $I S$

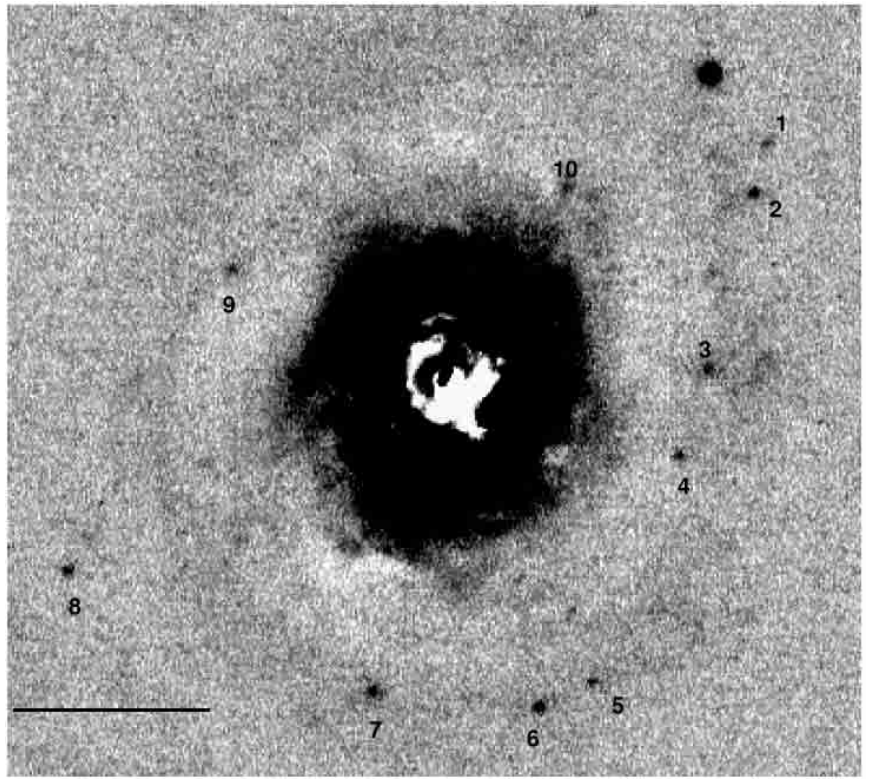

Fig. 3. $R$ image of NGC 5666 minus bulge+disk model galaxy. North is on top and East to the left. The lower left bar is $10^{\prime \prime}(=1.5 \mathrm{kpc})$ long.

versus $m$ function (Sérsic 1982). Both methods show very similar results and they give $B_{\mathrm{t}}=13.75$, which is in good agreement with the value $\left(B_{\mathrm{t}}=13.80\right)$ listed in the RC3 Catalogue (de Vaucouleurs et al. 1991). The derived asymptotic color is $(B-R)_{\mathrm{t}}=1.03$.

Table 2 lists the photometric parameters obtained for the bulge, the disk and the galaxy as a whole. The effective and central surface brightnesses are $m_{\mathrm{e}}=-2.5 \log \left(I_{\mathrm{e}}\right)$ and $m_{0}=$ $-2.5 \log \left(I_{0}\right)$, and are expressed in mag $\operatorname{arcsec}^{-2}$. The bulge-todisk ratios were derived from the luminosities calculated above (Eqs. (5) and (6)). Throughout this paper we have adopted a Hubble constant $H_{0}=70 \mathrm{~km} \mathrm{~s}^{-1} \mathrm{Mpc}^{-1}$.

The luminosity profiles of NGC 5666 in $B$ and $R$ are difficult to interpret, since, as pointed out by LSG87, they closely follow the $R^{1 / 4}$ law beyond $r>5^{\prime \prime}$, despite the fact that this is a spiral galaxy, as attested by the spiral structure (Fig. 2) and the gas contents (see Sect. 5). The bulge and the disk components are atypical of a late-type galaxy: the bulge has a low $m_{\mathrm{e}}$ and is very extended and shallow $\left(r_{\mathrm{e}}=13^{\prime \prime}\right.$, which corresponds to $1.9 \mathrm{kpc}$ ), while the disk has a very high $m_{\mathrm{o}}$ and steep profile (with a scale-length $d_{1}=4^{\prime \prime}$, which corresponds 
Table 3. Photometry of the resolved HII regions.

\begin{tabular}{rccr}
\hline \hline ID & radius ["] & $m_{B}$ & $(B-R)$ \\
\hline 1 & 0.3 & 19.23 & 0.05 \\
2 & 0.3 & 19.63 & -0.02 \\
3 & 0.5 & 18.58 & -0.19 \\
4 & 0.3 & 19.50 & 0.31 \\
5 & 0.3 & 18.73 & -0.31 \\
6 & 0.3 & 19.42 & 0.08 \\
7 & 0.3 & 19.41 & 0.30 \\
8 & 1.1 & 16.50 & -0.24 \\
9 & 0.5 & 18.22 & -0.44 \\
10 & 0.3 & 19.13 & -0.35 \\
11 & 0.6 & 17.71 & -0.28 \\
12 & 0.3 & 19.60 & 0.33 \\
13 & 0.3 & 19.61 & 0.28 \\
14 & 0.6 & 18.15 & -0.31 \\
15 & 0.6 & 17.75 & -0.36 \\
16 & 0.5 & 19.87 & -0.30 \\
17 & 0.6 & 18.07 & -0.25 \\
\hline
\end{tabular}

to $0.6 \mathrm{kpc}$ ). The characteristics of the disk are also reminiscent of those of early-type galaxies such as those studied by Scorza et al. (1998).

If we compare these photometric parameters with those found in the literature for large samples of normal galaxies, we find the following. The disk central surface brightness $\left(m_{\mathrm{o}}\right)$ of NGC 5666 is higher than those of the 86 galaxies in de Jong's (1996) sample, and makes it a type $t=4$ or less. On the other hand, the bulge effective surface brightness $\left(m_{\mathrm{e}}\right)$ is normal for a late-type galaxy according to de Jong (1996, his Fig. 10).

The photometric properties of the bulge and disk of NGC 5666 also go in opposite ways to those of the normal spirals of Márquez \& Moles (1999), whose bulges are compact and have on average small effective radii $\left(r_{\mathrm{e}}^{B}=0.22 \pm 0.65 \mathrm{kpc}\right)$ and high effective surface brigthnesses $\left(m_{\mathrm{e}}^{B}=20.10 \pm 0.54\right)$, while disks are more diffuse and extended, with lower central surface brightnesses $\left(m_{0}^{B}=20.9 \pm 0.6\right)$ and larger scale lengths $\left(d_{l}^{B}=3.0 \pm 1.5 \mathrm{kpc}\right)$. Furthermore, the $B / T$ parameter (defined by Kent 1985) for NGC 5666 is 0.4 , a value which is typical of Sa galaxies.

Finally, the concentration index $c_{31}$ defined by de Vaucouleurs (1977) has a value of 2.5, which corresponds to an Sc+ galaxy (Pérez-González et al. 2001, their Table 7). This is the only photometric parameter in agreement with the classification of our galaxy. Since this parameter does not depend on the photometric decomposition into bulge and disk, this suggests that the laws adopted by us for this decomposition are not valid in the case of NGC 5666, and/or that it is a rather peculiar spiral galaxy.

\section{Detailed morphology}

The next step was to build galaxy models from the two profiles described in Table 2 and to subtract them from the real images. The result is shown in Figs. 2 and 3 for the inner ( $B$ image) and the outer region ( $R$ image) respectively.
Two main structures appear: a very conspicuous spiral pattern (Fig. 2) that is limited to the plateau of the luminosity profile, i.e. $2^{\prime \prime} \leq r \leq 5^{\prime \prime}$, and what looks like a faint and smooth tidal tail (Fig. 3). These two subsystems are responsible for the two excesses in the luminosity profiles.

The inner disk shows a patchy spiral structure, dust lanes and a number of strong HII regions, an appearance which is typical of late-type galaxies. One notable difference is that, in normal late-type galaxies, the spiral structure extends over the whole disk. Table 3 lists the photometry of the resolved HII regions, whose identification numbers appear in Fig. 2. The $B$ magnitudes and $(B-R)$ colors were extinction corrected using $E(B-V)=0.4$ (see Sect. 5.3) and $E(B-R)=0.71$. This value was calculated using the extinction curve from Savage $\&$ Mathis (1979).

There are two categories of HII regions according to color: those with $(B-R) \sim-0.2 \pm 0.2$ and those with $(B-R) \sim$ 0.3 . This effect could be indicative of the presence of young and old star forming regions or just be the consequence of dust effects. We also note that the bluer regions are on average one magnitude brighter than the redder ones. In particular, region 8 (hereafter HII-8, for which we have the spectrum, see Sect. 5) has $m_{B}=16.50$, which corresponds to $M_{B}=-16$.

The faint and smooth (presumed tidal) feature in the outer region rises at a radius of about $15^{\prime \prime}$ in the NW direction and it wraps around the whole galaxy. This feature is dotted with small knots about $0.3^{\prime \prime}(=45 \mathrm{pc})$ in radius. This characteristic is typical of interacting or merging galaxies which show tidal tails containing a variety of stellar structures, with sizes ranging from those of globular clusters up to those of dwarf galaxies (Weilbacher et al. 2003). The absolute magnitudes for these features are in the range $M_{R}=-9$ to -10 . Most features are likely to be star clusters, except knot 1 which is very elongated, and knots 3, 5, 7 and 10, which are too blue; knot 7 in particular is very blue, with $(B-R)=0.06$. The latter may be very young star clusters formed in the recent starburst (see Sect. 5), or super-star clusters like those of NGC 1741 (Johnson et al. 1999).

\section{The gas and stellar populations of NGC 5666}

\subsection{The ionized gas and stellar populations}

The spectrum of the nuclear region of NGC 5666 is presented in Fig. 4a. It was extracted from a rectangular aperture centered at the peak of the brightness profile and the corresponding size is $3^{\prime \prime}(\sim 0.45 \mathrm{pc})$ in radius. It corresponds to an $\mathrm{Sb}$ type galaxy (Kennicutt 1991). The spectrum shows a continuum dominated by evolved giants stars and the additional signatures of a younger stellar population are more evident than in a typical Sa type spectrum. The $\mathrm{H} \alpha$ and [NII] $\lambda \lambda 6548$, 6584 emission lines are prominent and the equivalent width is $E W(\mathrm{H} \alpha+[\mathrm{NII}]) \sim 30 \AA$. This value is typical for normal isolated spirals (Kennicutt \& Kent 1983). No forbidden oxygen lines were detected, meaning that this galaxy is rather metal rich.

Figure $4 \mathrm{~b}$ shows a spectrum of HII- 8 , which is located $3^{\prime \prime}$ west of the nucleus (see Table 3 ). This spectrum shows a weak 

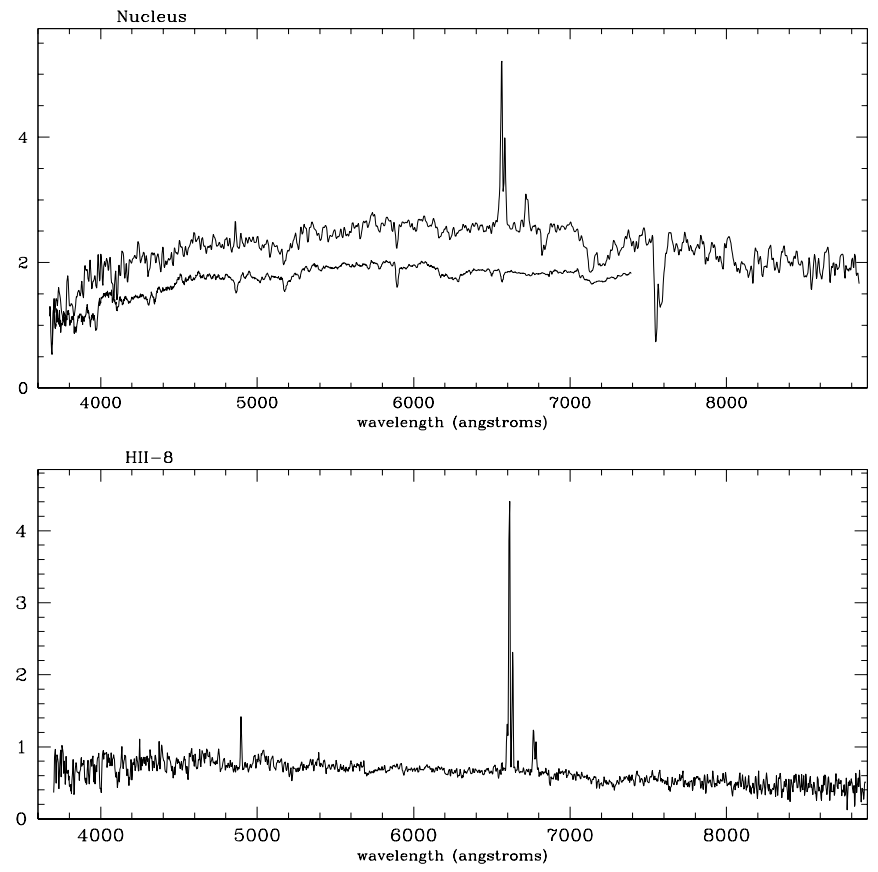

Fig. 4. Spectra of the nucleus (top) and of HII-8 (bottom). The combined template formed with $80 \%$ of E1 and $20 \%$ of S7 is also shown. A constant have been added to the template for ease visualisation. The plots show flux in units of $10^{-15} \mathrm{erg} \mathrm{cm}^{-2} \mathrm{~s}^{-1} \AA^{-1}$. The size of the region extracted is $\sim 0.45 \mathrm{pc}$.

continuum and the same emission lines as in the nuclear spectrum, although with stronger fluxes and larger $E W \mathrm{~s}$.

We have used the $E W$ of the more prominent absorption

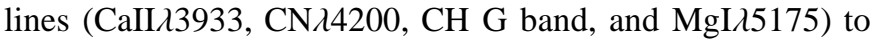
characterize the stellar population of NGC 5666. The $E W \mathrm{~s}$ were used to classify the spectrum in terms of Bica's (1988) templates, which span the properties of the more usual nuclear stellar populations observed in normal galaxies. In general terms the characteristics of the templates are the following: E1-E3 and S1-S3 are red stellar populations with metallicity decreasing from 4 to 1 times solar. S4-S7 is a sequence of increasing contributions from the blue components, $\mathrm{S} 4$ contains $10 \%$ of flux contribution at $\lambda 5870 \AA$ from populations younger than 1 Gyr, while S7 contains $80 \%$. However, we could not fit in a satisfactory way both the continuum slope and the $E W$ of the absorption features with a single stellar population. We thus had to find a suitable combination of Bica's templates to account for the main properties of NGC 5666's stellar populations. Figure $4 \mathrm{a}$ shows the results. A template formed with $80 \%$ of E1 and $20 \%$ of S7 fits the stellar spectrum quite well. This new template was then subtracted from the original galaxy spectrum in order to get the pure emission-line spectrum of the nucleus. Table 5 lists the fluxes and the $E W$ s of the detected emission lines as well as the internal extinction $E(B-V)$. This value was calculated assuming an intrinsic $\mathrm{H} \alpha / \mathrm{H} \beta$ ratio of 2.85 .

The main differences between the emission-line spectra of the nucleus and of HII- 8 lie in the [SII] $\lambda 6717 /[$ SII] $\lambda 6731$ ratio and reddening. Region HII- 8 has a reddening which is almost double that of the nucleus, and a ratio [SII] $\lambda 6717 /[$ SII] $]$ 6731> 1 , which indicates low electronic densities, as expected in normal HII regions.
Table 4. Photometry of the outer knots.

\begin{tabular}{rcccr}
\hline \hline ID & radius ["] & $m_{B}$ & $m_{R}$ & $(B-R)^{a}$ \\
\hline 1 & 0.3 & $\ldots$ & 23.50 & $>1.0$ \\
2 & 0.3 & $\ldots$ & 22.71 & $>1.8$ \\
3 & 0.3 & 23.47 & 22.84 & 0.63 \\
4 & 0.3 & $\ldots$ & 22.99 & $>1.5$ \\
5 & 0.3 & 23.77 & 23.25 & 0.52 \\
6 & 0.3 & 23.82 & 22.51 & 1.31 \\
7 & 0.3 & 22.75 & 22.69 & 0.06 \\
8 & 0.3 & $\ldots$ & 22.91 & $>1.6$ \\
9 & 0.3 & $\ldots$ & 23.27 & $>1.2$ \\
10 & 0.3 & 23.72 & 22.99 & 0.73 \\
\hline
\end{tabular}

${ }^{a}$ Lower limit calculated from the limiting magnitude in $B$ $\left(m_{B}=24.5\right)$.

\subsection{Age and metallicity of the burst}

The nucleus and HII- 8 have rather similar metallicities. The latter were estimated using the N2 calibrator (Denicoló et al. 2002) which is very useful in the absence of the forbidden oxygen lines. Both spectra give $12+\log (\mathrm{O} / \mathrm{H})=8.9$, which corresponds to solar metallicity (the solar value is 8.91 ; Denicoló et al. 2002). We can then determine the age of the burst of star formation, using the models of Cerviño \& Mas-Hesse (1994) which give the age of the burst as function of $E W(H \beta)$ for a wide range of metallicities. In the present case the burst ages in the nucleus and in HII- 8 are 8 and 6 Myr respectively, provided that the main source of ionization is OB stars and that no additional nonthermal ionizing sources, an active galactic nucleus or shock-heated gas, are present. We also verified that this is the case, using the $([\mathrm{SII}] \lambda 6717+[\mathrm{SII}] \lambda 6731) / \mathrm{H} \alpha$ and [NII] $\lambda 6584 / \mathrm{H} \alpha$ ratios (Coziol et al. 1997).

\subsection{Dust}

This is an IRAS-bright galaxy, where dust is assumed to be at the origin of the far-infrared emission. The presence of young hot stars (attested by the Balmer recombination lines) shows that there is warm dust, in addition to the cold dust heated by old red stars. The young stars are distributed in the inner disk with spiral structure, and the $(B-R)$ profile indicates that the reddening due to dust occurs mostly in that region.

The total apparent magnitude within this inner disk is 14.60. The correction for internal extinction, using $E(B-V)=$ 0.40 obtained in Table 5, gives a corrected magnitude of 12.85 . Adding the corresponding luminosity to that of the outer region (presumably unaffected by extinction) leads to a corrected total apparent magnitude of 12.62. We also correct by 0.11 for galactic extinction (Schlegel et al. 1998), but not for inclination effect, because this is a peculiar galaxy, with a disk of unknown thickness. The corresponding absolute magnitude is $M_{B}=-20.11$, which agrees very well with the value derived from the Tully-Fisher relation (Sect. 5.4). The metallicityluminosity relation (Zaritsky 1993), together with the metallicity derived in Sect. 5.2, also suggests an absolute magnitude of 
Table 5. Emission line fluxes ${ }^{a}$. and equivalent widths ${ }^{b}$

\begin{tabular}{|c|c|c|c|c|c|c|c|c|c|c|c|}
\hline \multirow[b]{2}{*}{ Spectrum } & \multicolumn{2}{|c|}{$\mathrm{H} \alpha$} & \multicolumn{2}{|c|}{$\mathrm{H} \beta$} & \multicolumn{2}{|c|}{$[\mathrm{NII}] \lambda 6584$} & \multicolumn{2}{|c|}{$[\mathrm{SII}] \lambda 6717$} & \multicolumn{2}{|c|}{$[\mathrm{SII}] \lambda 6731$} & \multirow[b]{2}{*}{$E(B-V)$} \\
\hline & Flux & $E W$ & Flux & $E W$ & Flux & $E W$ & Flux & $E W$ & Flux & $E W$ & \\
\hline nucleus & 40.0 & 14.4 & 9.7 & 3.7 & 19.2 & 6.9 & 5.6 & 2.0 & 6.7 & 2.4 & 0.40 \\
\hline HII-8 & 36.0 & 53.8 & 6.0 & 8.7 & 16.0 & 23.5 & 7.4 & 11.1 & 3.7 & 5.5 & 0.70 \\
\hline
\end{tabular}

${ }^{a}$ In units of $10^{-15} \mathrm{erg} \mathrm{cm}^{-2} \mathrm{~s}^{-1}$.

${ }^{b}$ In $\AA$.

that order. This is thus a giant galaxy, but a very compact one, since the isophotal radius at $m_{B}=25 \mathrm{mag} \mathrm{arcsec}-2$ is about $29^{\prime \prime}$, or $4.5 \mathrm{kpc}$. The ratio $L_{\mathrm{FIR}} / L_{B}=0.39$ makes it a very moderate IRAS-bright galaxy. One possible reason, which will appear in the next section, is that the far infrared luminosity is limited to the inner disk of the galaxy.

\subsection{The neutral gas}

The HI in NGC 5666 is more extended than the light distribution by a factor of 3 and oriented in approximately the same direction. Its distribution is clumpy, with the overall appearance of a ring, and there is a hint of a warp in the outer regions (LSG87). The ratio $M_{\mathrm{HI}} / L_{B}$ is 0.14 , typical for an S0aSa galaxy (Roberts \& Haynes 1994). The rotation curve rapidly reaches a plateau, at about $150 \mathrm{~km} \mathrm{~s}^{-1}$, which, with an inclination of $29^{\circ}$ gives a corrected velocity of rotation of $164 \mathrm{~km} \mathrm{~s}^{-1}$. The absolute magnitude predicted by the Tully-Fisher relation (Fouqué et al. 1990) is -20.1. This value is in perfect agreement with our estimate from the photometry (Sect. 5.3).

We have also calculated $M / L_{B}$, which reaches a value of 6 at a radius of $9 \mathrm{kpc}$ : this is more typical of ellipticals than spirals.

$\mathrm{CO}$ has also been detected in this galaxy (Wiklind et al. 1995; Young 2002). The CO rotation curve predicts that $M / L_{B} \sim 2$ in the inner $2.4 \mathrm{kpc}$. The ratios $L_{\mathrm{FIR}} / M\left(\mathrm{H}_{2}\right)=9.4$ and $L_{B} / M\left(\mathrm{H}_{2}\right)=24.1$ were derived using the molecular masses estimated by Wiklind et al. (1995); while the first one is very close to the typical value for spirals $(\sim 9)$, the second one is much too high for this classification $(\sim 7)$. This indicates that $\mathrm{CO}$ and far infrared luminosity (thus dust) are confined in the same inner region. In contrast, elliptical galaxies show on average $L_{\mathrm{FIR}} / M_{\mathrm{H}_{2}} \sim 22$ and a $L_{B} / M_{\mathrm{H}_{2}} \sim 230$.

Both the $\mathrm{HI}$ and $\mathrm{CO}$ rotation curves show that the neutral gas is in circular rotation, in other words in dynamical equilibrium. Finally, the ratio $M\left(\mathrm{H}_{2}\right) / M(\mathrm{HI})=0.53$.

\section{Discussion}

The spiral nature of this galaxy is borne out by our photometric and spectroscopic data. The spiral structure suggests a latetype galaxy. The luminosity profile is not a simple $R^{1 / 4}$ law and can be decomposed into a bulge and a disk component. The concentration index $c_{31}$ is that of an $\mathrm{Sc}+$ galaxy. The equivalent width of $\mathrm{H} \alpha+\mathrm{NII}$ is typical of spiral galaxies. The neutral (HI and $\mathrm{CO}$ ) gas contents also point to a spiral galaxy.
However, this is not a normal Sc-Sd galaxy for several reasons.

- The underlying stellar population could not be fitted in terms of Bica's templates for normal spiral galaxies, but rather with elliptical (E1) + very young (S7) stellar populations.

- The spiral arms are confined to the inner sixth of the galaxy.

- The bulge is extended and the disk has a small scale length.

- The B/T ratio (Kent 1985) is typical of Sa galaxies.

- The $M_{\mathrm{HI}} / L_{B}$ and $M_{\mathrm{H}_{2}} / L_{B}$ ratios are those of an early-type galaxy.

- There is a faint and probably tidal feature dotted with small bright knots ( $45 \mathrm{pc}$ in radius).

In order to reconcile these contradictory properties, we suggest that NGC 5666 is a minor merger, where a small earlytype disk galaxy accreted a gas-rich dwarf galaxy. Numerical simulations by Walker et al. (1996) provide several interesting predictions, some of which could be tested: the disk survives, but thickens and gets hotter (it has had time to cool again, since the galaxy appears to be rotationally supported); a larger bulge forms (which is still partly obscured by dust), warps arise (which seems to be the case for the HI distribution). Simulations of stochastic self-propagating star formation also make interesting predictions, although they do not identify the origin of the spiral structure. Gerola \& Seiden (1978) show a model where star formation travels from center to edge in about 3 Gyr. In NGC 5666, it has only traveled one sixth of the way, thus could have started about $500 \mathrm{Myr}$ ago. This would date the merger event. Other possible effects of the encounter have been identified in Sect. 4, namely small knots in the outer disk and a large and smooth tidal feature in the outer regions.

\section{Conclusion}

We have presented new optical CCD images and spectra of the galaxy NGC 5666, previously thought to be an elliptical. On our images appear a very conspicuous spiral arm pattern and dust patches that were not noticed by previous authors due to the insufficient resolution of their images. However, the photometric analysis indicates that this is a rather unusual spiral galaxy: it does not have a standard bulb+disk morphology, it has a very small radius for its luminosity, and its spiral pattern is confined to the inner region. While most of its properties are those of a spiral, others are reminiscent of an elliptical, and there is an outer feature resembling a tidal tail. We are thus lead 
to interpret this galaxy as the result of a recent minor merger. If this is the case, the properties of this galaxy will be very useful to constrain the theory of hierarchical galaxy formation and to guide numerical investigations of the evolution of galaxies; to our knowledge, no predictions about the formation and evolution of spiral structure in gas-rich mergers have yet been published.

Recent analysis of the HDF showed that galaxies tended to be of smaller radius and higher surface brightness in the past, and mergers seems to be the mechanism (Ferguson et al. 2000). Thus, this galaxy can be a present day equivalent of high compact SB galaxies or can also be considered as a zero-redshift prototype of merger at an intermediate stage and should be very useful to interpret future observations of galaxies at large redshifts where mergers are known to be more frequent.

Acknowledgements. We thank P. Poulain and G. Monnet for obtaining the CCD images of this galaxy, and Ph. Prugniel and Dr. B. Binggeli (referee) for helpful comments and sugestions.

CJD is grateful with Paradies, Freiburg, and for the skilled and goodhumored assistance of the CASLEO staff.

\section{References}

Bica, E. 1988, A\&A, 195, 76

Cerviño, M., \& Mas-Hesse, J. M. 1994, A\&A, 284, 749

Coziol, R., Contini, T., Davoust, E., \& Considère, S. 1997, ApJ, 481, L67

de Jong, R. S. 1996, A\&A, 313, 45

Denicoló G., Terlevich, R., \& Terlevich, E. 2002, MNRAS, 330, 69

de Vaucouleurs, G. 1977 ApJS, 33, 211

de Vaucouleurs, G., \& Pence, W. D. 1979, ApJS, 39, 49

de Vaucouleurs, G., de Vaucouleurs, A., Corwin H. G. I. et al. 1991, The Third Reference Catalogue of Bright Galaxies (New York: Springer Verlag) (RC3)

Djorgovski, S. B. 1985, Ph.D. Thesis, California University, Brekeley Ferguson, H. C., Dickinson, M. \& Williams, R. 2000, ARA\&A, 38, 667
Fouqué, P., Bottinelli, L., Goughenheim, L., \& Paturel, G. 1990, ApJ, 349,1

Gerola, H., Seiden, P.E., 1978, ApJ, 223, 129

Gordon, M. A. 1991, ApJ, 371, 563

Huchtmeier, W. K. 1994, A\&A, 286, 389

Huchtmeier, W. K., Sage, L. J., \& Henkel, C. 1995, A\&A, 300, 675

Huchtmeier, W. K., \& Richter, O.-G. 1988, A\&A, 203, 237

Jedrzejewski, R. I., 1987, MNRAS, 226, 747

Johnson, K. E., Vacca, W. D., Leitherer, C., Conti, P. S., \& Lipscy, S. J. 1999, AJ, 117, 1708

Kennicutt, R. C. 1991, ApJS, 79, 255

Kennicutt, R. C., \& Kent, S. M. 1983, AJ, 88, 1094

Kent, S. M. 1985, ApJS, 59, 115

Lake G., Schommer, R. A., \& Gorkom, J. H. 1987, ApJ, 314, 57 (LSG87)

Lake G., \& Schommer, R. A. 1984, ApJ, 280, 107

Lees, J. F., Knapp, G. R., Rupen, M. P., \& Phillips, T. G. 1991, ApJ, 379,177

Márquez, I., \& Moles M. 1999, A\&A 344, 421

Nilson, P. 1973, Uppsala General Catalogue of Galaxies, Nova Acta Regiae Societatis Scientarium Upsaliensis, Ser. VA. vol. 1 (UGC)

Pérez-González, P. G., Gallego, J., Zamorano, J., \& Gil de Paz, A. 2001, A\&A, 365, 370

Peterson, S. D. 1979 ApJS, 40, 527

Prugniel, Ph., \& Heraudeau, P. 1998, A\&AS, 128, 299

Roberts, M. S., \& Haynes, M. P. 1994, ARA\&A, 32, 115

Savage, B.D., \& Mathis, J. S. 1979, ARA\&A, 17, 73

Sérsic, J. L. 1968, Atlas de galaxias australes, Observatorio Astronómico de Córdoba

Sérsic, J. L. 1982, Extragalactic Astronomy (Dordrecht: Reidel)

Schombert, J. M., \& Bothun, G. D. 1987, AJ, 93, 60

Schlegel, D. J., Finkbeiner, D. P., \& Davis, M. 1998, ApJ, 500, 525

Scorza, C., Bender, R., Winkelmann, C., Capaccioli, M, \& Macchetto, D. F. 1998, A\&AS, 131, 265

Stone, R. P. S. \& Baldwin, J. A. 1983, MNRAS, 204, 347

Walker, I. R., Mihos, J. C., Hernquist, L. 1996, ApJ, 460, 121

Weilbacher, P. A., Duc, P. A., \& Fritze-v. Alvensleben, U. 2003, A\&A, 397,545

Wiklind, T., Combes, F., \& Henkel, C. 1995, A\&A, 297, 643

Young, L. M. 2002, AJ, 124, 788

Zaritsky, D. 1993, PASP, 1006 